\title{
Chlorosis seedling lethality 1 encoding a MAP3K protein is essential for chloroplast development in rice
}

\author{
Jiayan Liang ${ }^{\dagger}$, Qiuxin Zhang ${ }^{\dagger}$, Yiran Liu, Jingjing Zhang, Wenyi Wang ${ }^{*}$ and Zemin Zhang ${ }^{*}$
}

\begin{abstract}
Background: Mitogen-activated protein kinase (MAPK) cascades are conserved signaling modules in eukaryotic organisms and play essential roles in immunity and stress responses. However, the role of MAPKs in chloroplast development remains to be evidently established.

Results: In this study, a rice chlorosis seedling lethality 1 (cs/1) mutant with a Zhonghua11 (ZH11, japonica) background was isolated. Seedlings of the mutant were characterized by chlorotic leaves and death after the trefoil stage, and chloroplasts were observed to contain accumulated starch granules. Molecular cloning revealed that OsCSL1 encoded a MAPK kinase kinase22 (MKKK22) targeted to the endoplasmic reticulum (ER), and functional complementation of OsCSL1 was found to restore the normal phenotype in cs/1 plants. The CRISPR/Cas9 technology was used for targeted disruption of OsCSL1, and the OsCSL1-Cas9 lines obtained therein exhibited yellow seedlings which phenocopied the cs/1 mutant. CSL1/MKKK22 was observed to establish direct interaction with MKK4, and altered expression of MKK1 and MKK4 was detected in the cs/1 mutant. Additionally, disruption of OsCSL1 led to reduced expression of chloroplast-associated genes, including chlorophyll biosynthetic genes, plastid-encoded RNA polymerases, nuclearencoded RNA polymerase, and nuclear-encoded chloroplast genes.
\end{abstract}

Conclusions: The findings of this study revealed that OsCSL1 played roles in regulating the expression of multiple chloroplast synthesis-related genes, thereby affecting their functions, and leading to wide-ranging defects, including chlorotic seedlings and severely disrupted chloroplasts containing accumulated starch granules.

Keywords: Rice, OsCSL1, Chloroplast development, MAP3K protein, Chloroplast-associated genes

\section{Background}

Chloroplasts are defined as organelles that play specific roles in the conversion of light energy to chemical energy via photosynthesis [1], and their functional and structural integrity are vital for normal plant growth and development $[2,3]$. Abnormal chloroplast function is generally

\footnotetext{
*Correspondence: wywang@scau.edu.cn; zmzhang@scau.edu.cn †Jiayan Liang and Qiuxin Zhang contributed equally to this work. State Key Laboratory for Conservation and Utilization of Subtropical Agro-Bioresources, Guangdong Provincial Key Laboratory of Plant Molecular Breeding, College of Agriculture, South China Agricultural University, Guangzhou 510642, China
}

reflected in changes in leaf pigmentation $[4,5]$. In rice, several pentatricopeptide repeat proteins (PPR) have been identified and are reportedly involved in chloroplast development, including Young Seedling Albino (YSA) [6], Albino Seedling Lethality3 (ALS3) [7], Thermo-sensitive Chlorophyll-Deficient 10 (TCD 10) [8], and OsPPR6 [9]. Additionally, genes from several other families have been implicated in chloroplast development. For example, Albino Leaf 1 (AL1) and AL2 encoding an octotricopeptide repeat protein and chloroplast group IIA intron splicing facilitator were identified, respectively [10, 11]; additionally, alterations in the number and structure of thylakoids resulted in the development of albino leaves 
and in the occurrence of seedling death at an early developmental stage in rice $[10,11]$. Furthermore, rice Young Leaf Chlorosis 2 (YLC2) has been shown to encode the stroma-localized heme oxygenase 2 , and seedlings of the $y l c 2$ mutant have been observed to exhibit a chlorotic phenotype and defective chloroplast structures [12]. Recently, Glycinamide ribonucleotide synthetase (GARS), which catalyzes the second step in purine nucleotide biosynthesis, has been identified to be involved in chloroplast development in rice by affecting the expression of plastid-encoded genes [13].

Chloroplasts are semi-autonomous organelles containing a unique genome and gene expression system [14]. Precise chloroplast function is coordinately mediated by two types of RNA polymerase, namely the nuclearencoded RNA polymerases (NEPs) and plastid-encoded RNA polymerases (PEPs) [15, 16], which are essential for the biogenesis of photosynthetically active chloroplast in plants $[16,17]$. The NEP complex is encoded by RPOTp and RPOTmp, the knockout of which has been found to result in delayed chloroplast biogenesis [18, 19]. Apart from PEPs and NEPs, chlorophyll biosynthetic genes (CBGs) and nuclear-encoded chloroplast genes (NECGs) have also been established to be involved in chloroplast development.

Mitogen-activated protein kinase (MAPK) cascades are considered important mechanisms involved in the transmission of exogenous or developmental signals to target molecules, and are generally highly conserved in eukaryotes $[20,21]$. These cascades are typically characterized by the sequential phosphorylation of MAPK kinase kinase (MKKK/MAP3K), MAPK kinase (MKK/MAP2K), and MAPK. In rice, MAPK signaling pathways have been shown be involved in eliciting defense responses against blight disease [22], rice blast [23], and brown planthopper infestation [24, 25], and also in the responses to multiple abiotic stimuli [26-30]. OsMPK6 has been identified as a negative regulator involved in the resistance to bacterial pathogens, the reduced expression of which enhances resistance to different races of Xanthomonas oryzae $\mathrm{pv}$. oryzae, mediated via salicylic acid and jasmonic acid signaling pathways [31, 32]. Recent studies have also revealed the involvement of MAPK cascades in plant development. For example, in rice, the OsMKKK10OsMKK4-OsMPK6 cascade has been established to positively control grain size and is negatively regulated by GRAIN SIZE AND NUMBER1 (GSN1) [33, 34]. Currently, however, much remains unknown regarding the role of MAPK cascades in chloroplast development.

In this study, characterization of a novel MAP3K gene involved in chloroplast development in rice was reported, designated chlorosis seedling lethality 1 (csl1), the mutation of which has been reported to result in energy deficiency and premature seedling death. OsCSL1 encodes a MAPK kinase kinase22 (MKKK22), which targets the endoplasmic reticulum (ER); furthermore, protein-protein interaction analyses revealed that OsCSL1/ OsMKKK22 interaction with MKK4. Expression of MKK4 was reduced in the csl1 mutant. Collectively, these observations indicate that the MKKK2-MKK4 signaling pathway potentially plays an important role in chloroplast development in rice.

\section{Results}

The cs/1 mutation results in the development of chlorotic seedlings and a lethal phenotype

To investigate the mechanisms underlying MAPK involvement in chloroplast development, a mutant, chlorosis seedling lethality 1 (csl1), displaying defects in leaf pigmentation was identified among rice T-DNA insertion populations with a Zhonghua11 (japonica) background. Compared with the green leaves of wild-type (WT) plants, the csl1 mutant exhibited a chlorotic seedling phenotype that developed prior to the trefoil stage (Fig. 1A, B). Unlike other chlorotic or albino leaf mutants, in which the leaf defects could be restored at later stages of development [19], csl1 seedlings gradually withered and eventually died. To determine whether the observed chlorosis was associated with altered photosynthetic efficiency, chlorophyll contents in leaf blades and sheaths at the trefoil stage were further evaluated. The contents of chlorophylls $a$ and $b$ and total chlorophyll content in both leaf blades and sheaths were significantly reduced in the $c s l 1$ mutant compared with WT seedlings (Fig. 1C), whereas no appreciable differences were detected between WT and heterozygous csll lines (Fig. S1).

Chloroplasts are indispensable for normal plant growth and development, not only as an essential site for photosynthesis but also for a wide range of biochemical processes, including the synthesis of pigments, lipids, and hormones. Considering that csl1 mutant seedlings were characterized by a yellow pigmentation and reduced chlorophyll contents, csl1 mutation exerted an influence on chloroplast development was investigated. Transmission electron microscopy (TEM) observations of the chloroplast structures in csl 1 and WT seedlings at the two-leaf stage revealed that WT chloroplasts could be characterized by a distinct unidirectional thylakoid structure, whereas the intracellular structure of $c s l 1$ mutant chloroplasts seemed to be disordered with indistinct thylakoids oriented in different directions, which accordingly contributed to a marked disruption of chloroplasts in the csl1 mutant (Fig. 1D). Moreover, pronounced accumulation of starch granules in chloroplasts of the csl1 mutant was detected (Fig. 1D). Collectively, these 


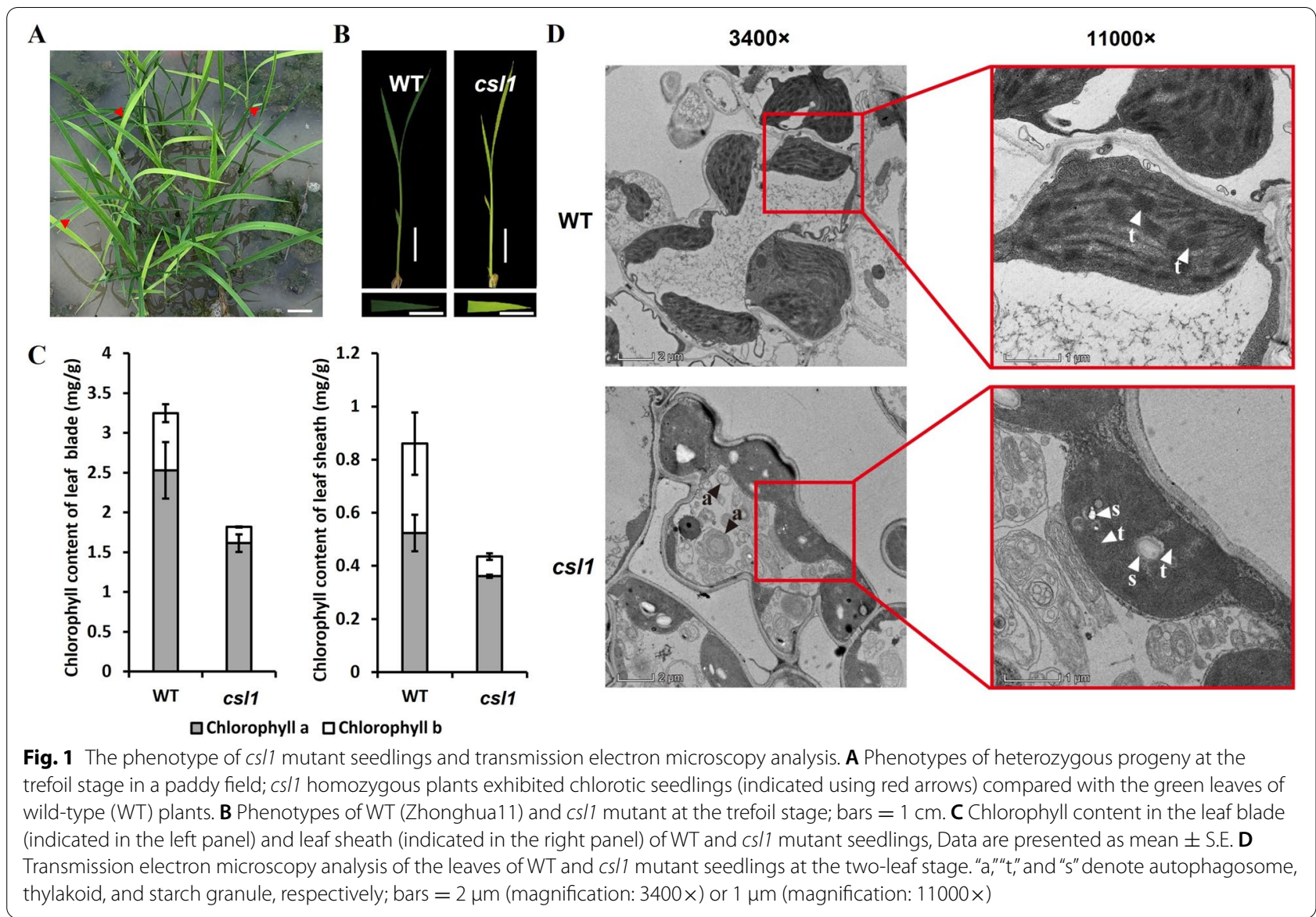

observations indicate that OsCSL1 is essential for normal chloroplast development in rice.

\section{Molecular cloning of OSCSL1}

Genetic analysis indicated that the $c s l 1$ phenotype could be subjected to control by a single recessive gene, as evidenced by the 3:1 segregation ratio between green and yellow seedlings $\left(x^{2}=0.107\right.$, Table S1). To further characterize this gene, inverse polymerase chain reaction (IPCR) was performed to isolate the T-DNA genomic flanking regions in the csl1 mutant (Fig. S2). A BLAST search (https://rapdb.dna.affrc.go.jp/) revealed that the sequences of the flanking region were similar to those of a gene (Os03g0703400) predicted to encode a MAPK kinase kinase22 (MKKK22) located on rice chromosome 3 , which has not been functionally characterized thus far. The coding region of Os03g0703400 contains eight exons and seven introns (Fig. 2A). Notably, the full-length OsCSL1 protein consists of 489 amino acids, as opposed to the predicted 654 residues found in databases, the difference between which is attributable to a 165-amino acid deletion at the beginning of the first exon in the amplified product (Fig. S3).
The site of the T-DNA insertion is located between bases +4214 and +4322 in the seventh intron, leading to a 109-bp segment deletion in Os03g0703400 (Fig. 2A). To eliminate interference from contiguous genes, expression levels of Os03g0703300, Os03g0703400 (MKKK22/ OsCSL1), and Os03g0703600 were examined (Fig. 2B). RT-qPCR analysis revealed no appreciable differences between WT and csl1 mutant plants with respect to the levels of Os03g0703300 and Os03g0703600 transcripts (Fig. 2C); however, the level of Os3g0703400 (OsCSL1) was found to be significantly reduced in the $c s l 1$ mutant compared with the WT (Fig. 2C). Collectively, these findings indicate that $\mathrm{Os} 3 g 0703400$ is a strong candidate for the $\operatorname{OsCSL1} 1$ gene.

\section{Mutation of $\operatorname{OsCSL1}$ is responsible for the development of a chlorotic seedling phenotype}

To verify whether the chlorotic seedling phenotype was associated with a defective Os03g0703400 (OsCSL1), genetic complementation assay using heterozygous csl1/OsCSL1 plants was performed, owing to the lethality of the csl1 mutation in homozygous seedlings. A $\sim 9-k b$ WT genomic DNA sequence, comprising a 


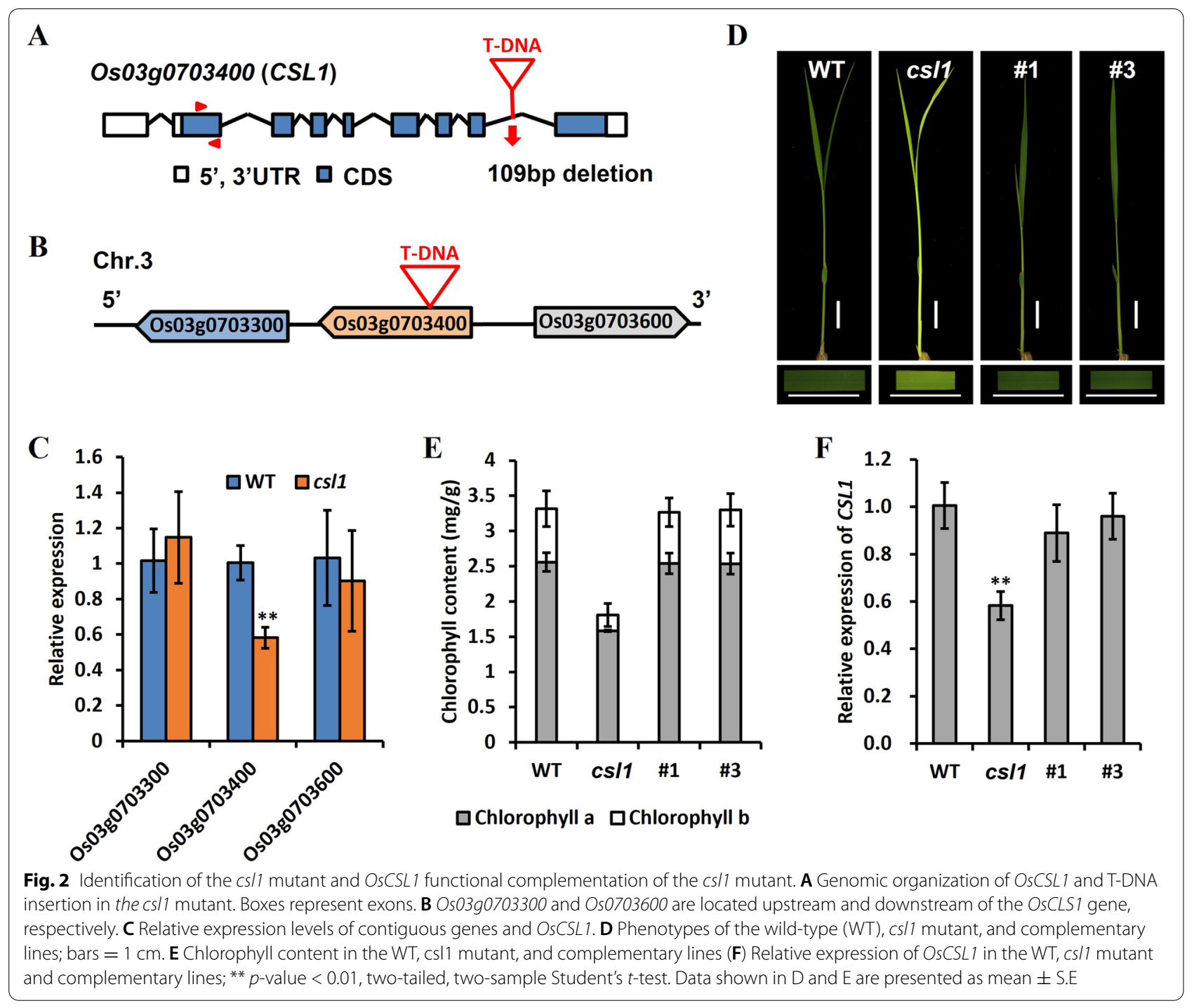

$\sim 2.5$ - $\mathrm{kb}$ sequence upstream of the start codon, a $\sim 5.5$ $\mathrm{kb}$ open reading frame, and a $\sim 1$-kb sequence downstream of the stop codon, was cloned into a binary vector and transformed into heterozygous csl1/OsCSL1 plants. Positive transgenic plants derived from the csl1/csl1 background were obtained, and used for further analyses. As shown in Fig. 2D, csl1 seedlings characterized by a defective chlorotic phenotype were rescued by the OsCSL1 transgene (Fig. 2D). Moreover, the expression of OsCSL1 and chlorophyll contents in the transgenic seedlings recovered to normal levels (Fig. 2E, F). These observations thus revealed that the chlorotic phenotype of csl1 mutant seedlings was associated with a defective Os03g0703400, thereby indicating that OsCSL1 was the causal gene and it played an essential role in the regulation of rice leaf pigmentation.
To further examine the molecular function of OsCSL1 in rice, OsCSL1 knockout mutant lines in the Zhonghua11 background were generated using the CRISPR/ Cas9 genome editing technique. Accordingly, a series of transgenic OsCSL1-Cas9 lines were generated using a target site located in the fifth exon (Fig. 3A). Subsequent phenotypic observations revealed that the seedlings of OsCSL1-Cas9 lines presented with a chlorotic phenotype prior to the trefoil stage (Fig. 3B), which was similar to that of the csl1 mutant. Consistently, similar to the findings documented with the csl1 mutant, chlorophyll contents (chlorophyll $a$, chlorophyll $b$, and total chlorophyll) in the OsCSL1-Cas9 line seedlings were reduced compared with those in the WT plants (Fig. 3C). Moreover, the findings revealed that deletion and/or substitution events occurred in the OsCSL1-Cas9 lines (Fig. 3D); for 
A

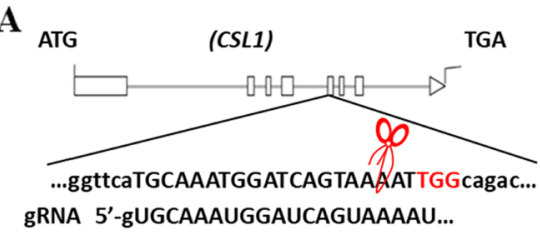

C

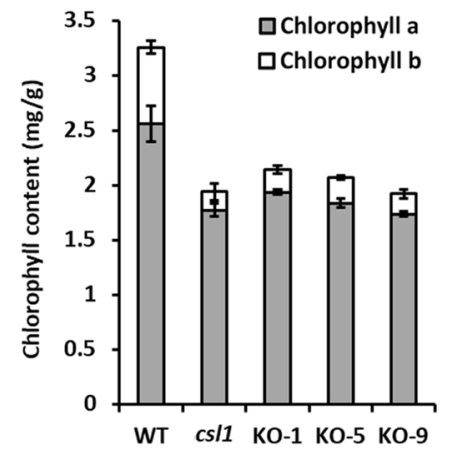

B

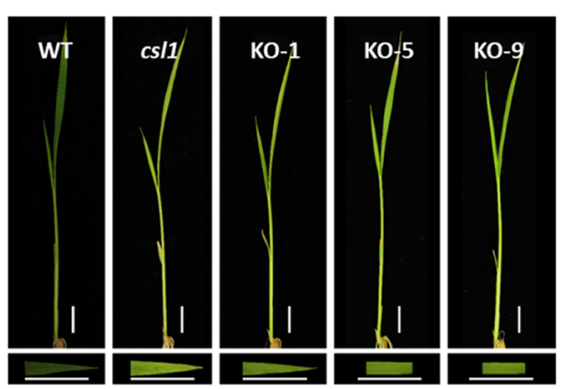

D

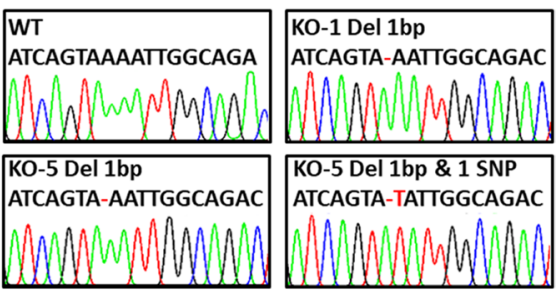

Fig. 3 Characterization of OSCSL1-Cas9 lines. A A schematic representation of knockout target sites of OsCSL1. B Phenotypes of the wild-type (WT), cs/1 mutant, and OsCSL1-Cas9 lines; bars = $1 \mathrm{~cm}$. C Chlorophyll content in the WT, cs/1 mutant, and OsCSL1-Cas9 lines. Data are presented as mean \pm S.E. D OsCSL1-Cas9 lines containing insertion and deletion mutations were confirmed via sequencing

example, OsCSL1-Cas9-1 and OsCSL1-Cas9-5, which contained a 1-bp deletion, and OsCSL1-Cas9-9, containing both deletions and substitutions (Fig. 3D). These findings indicated that OsCSL1 knockout plants phenocopied the T-DNA insertional mutant, and mutation of the OsCSL1 gene resulted in the development of the chlorotic seedling phenotype in rice.

\section{OsCSL1 is targeted to the endoplasmic reticulum}

To elucidate the temporal and spatial expression of OsCSL1, RT-qPCR to investigate the patterns of expression in different tissues was performed, including root, stem, leaf sheath, leaf blade, young panicle, and mature panicle. The results revealed that OsCSL1 was constitutively expressed in different tissues and organs, demonstrating markedly high levels in the leaf sheath and blade (Fig. 4A), and was weakly expressed in other tissues, such as the roots, stems, and young and mature panicles (Fig. 4A). These results thereby highlight the role of OsCSL1 in plant development, particularly, in the developmental stages in the leaves.

To examine the subcellular localization of OsCSL1, a CSL-GFP (green fluorescent protein) fusion protein was transiently expressed in rice protoplasts. An OsCSL1-GFP fusion protein under the control of the Zea mays ubiquitin promoter was assembled and subsequently transformed into rice protoplasts. Laser scanning confocal microscopy of the transformed protoplasts revealed an overlap of the green signals generated by OsCSL1-GFP, and the red signals derived from ER-mCherry [35], observed as an orange coloration (Fig. 4B), thus indicating that OsCSL1 was located in the ER.

\section{Altered expression of chloroplast-related genes is observed in the cs/1 mutant}

Considering abnormal chlorophyll metabolism and plastid development in the csl1 mutant, the expression of chloroplast development- and photosynthesisrelated genes were investigated, including chlorophyll biosynthetic genes (CBGs), plastid-encoded RNA polymerases (PEPs), nuclear-encoded plastid RNA polymerase (NEPs), and nuclear-encoded chloroplast genes (NECGs). In line with expectations, significant reductions in the expression levels of several CBGs in the csl1 mutant were detected, including those of OsHAP3, HemA, OsCAO, YGL1, and Cab1R, which were reduced by at least a $50 \%$ compared with those noted in the WT. Notable among these was the reduced expression of $O s H A P 3 A$ and $O s H A P 3 C$, which were barely detectable in the $\operatorname{csl} 1$ mutant (Fig. 5A). Furthermore, examination of the expression of PEPs, NEPs, and NECGs revealed that, with the exception of $p s b A$ and $p s b P$, the expression levels of all investigated genes were significantly impaired in the csl1 mutant (Fig. 5B-D). These findings accordingly provide evidence to indicate that chloroplast development, 


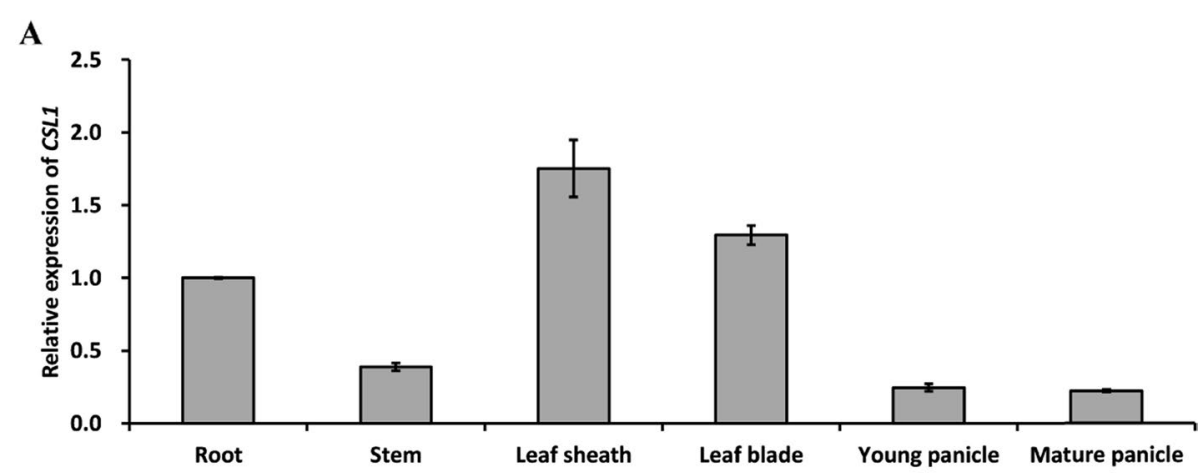

B

GFP

ER

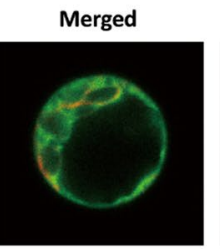

Bright Field
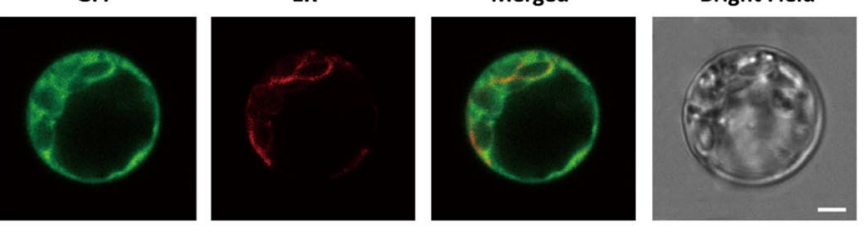

Ubi::CSL1-GFP
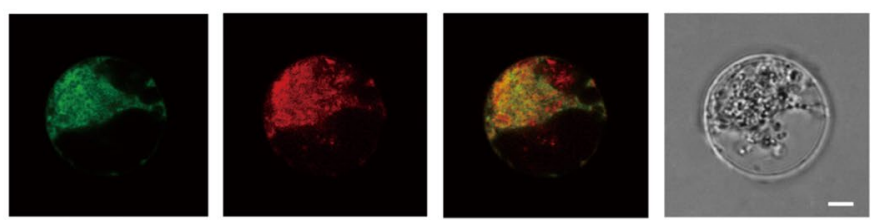

Fig. 4 Expression pattern of OsCSL1. A Expression levels in different organs. B Subcellular localization of the OsCSL1-GFP protein in rice protoplasts
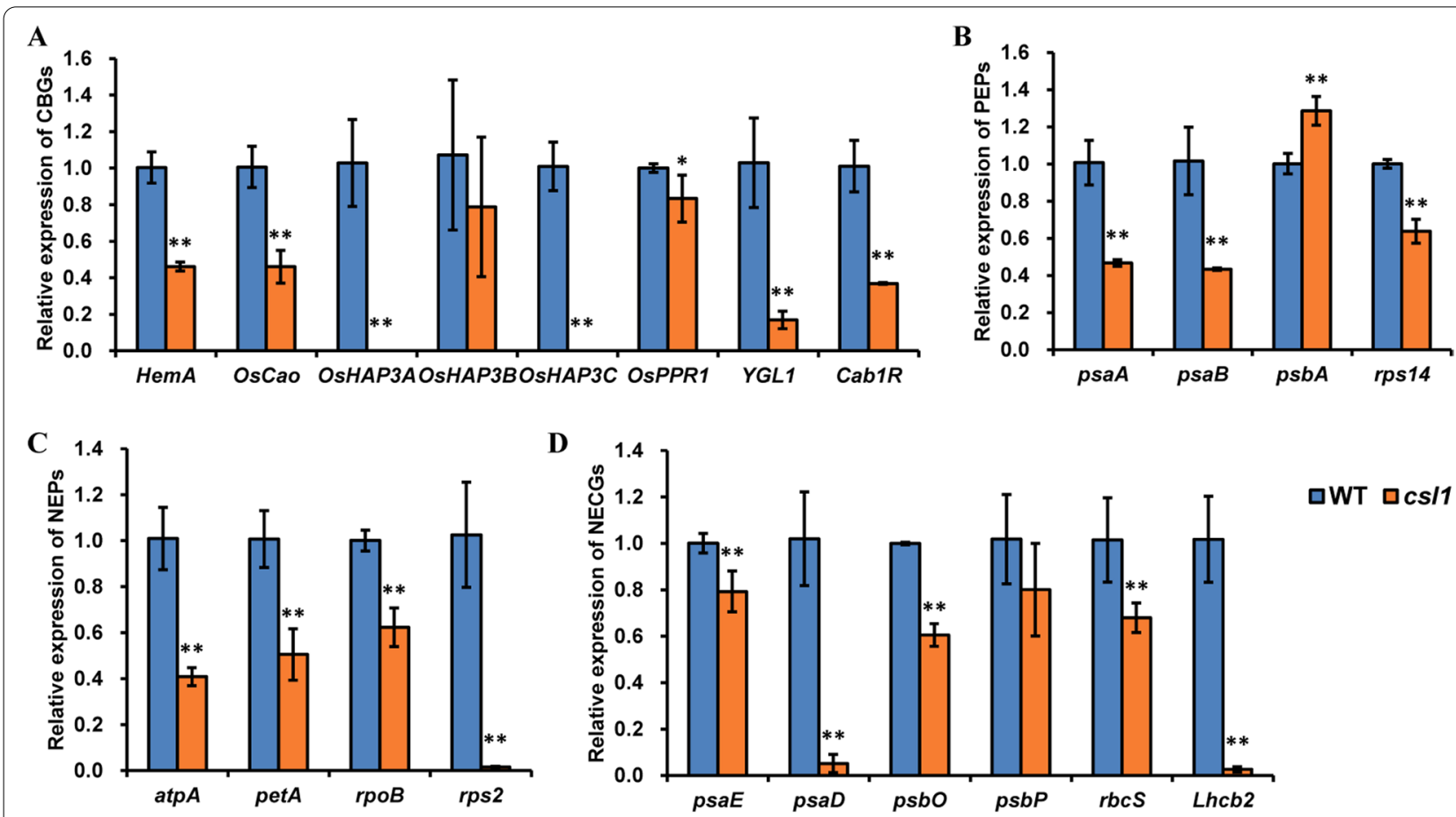

Fig. 5 Expression of chloroplast-associated genes. Relative expression of chlorophyll biosynthetic genes (CBGs) (A), plastid-encoded RNA polymerases (PEPS) (B), nuclear-encoded RNA polymerases (NEPS) (C), and nuclear-encoded chloroplast genes (NECGs) (D). Data are presented as mean \pm S.E., ${ }^{* *} p$-value $<0.01$, two-tailed, two-sample Student's $t$-test 


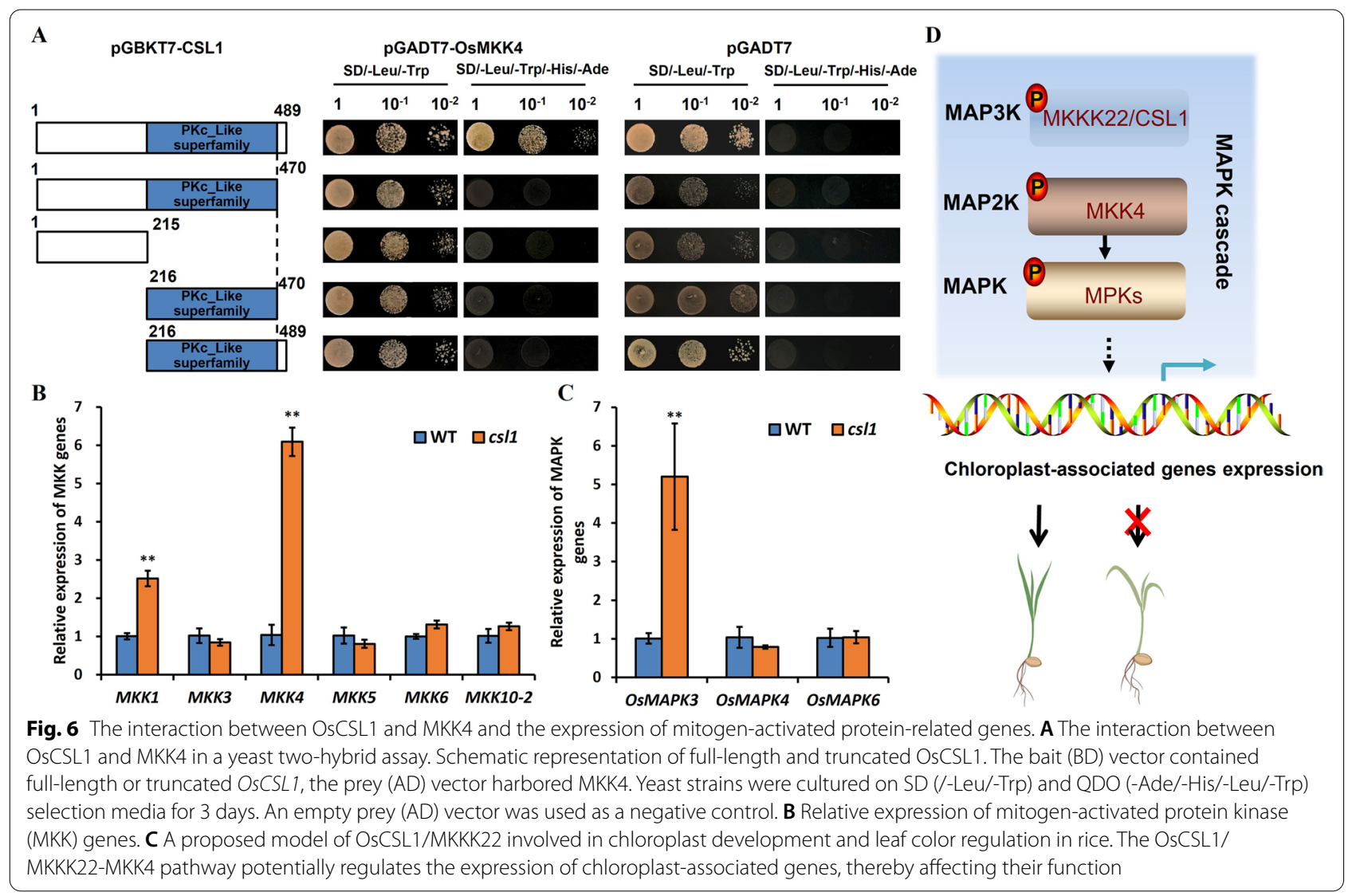

chlorophyll synthesis, and photosynthesis are potentially disrupted in the csll mutant.

\section{OsCSL1 establishes interaction with MKK4 and alters the expression of OsMKKs in the cs/1 mutant}

In the MAPK cascade, MAP3K catalyzes the phosphorylation of MKK proteins, which subsequently leads to the phosphorylation of MAPKs and results in the stimulation of downstream target genes. Previously, yeast two-hybrid $(\mathrm{Y} 2 \mathrm{H})$ and protein microarray analyses have revealed the interactions established between MKK and MPK pairs and among a range of MAPKs and potential substrates $[36,37]$. Thus, in the present study, $\mathrm{Y} 2 \mathrm{H}$ assays were used to examine the interactions between OsCSL1/ MKKK22 and MKKs, using either full-length or truncated OsCSL1 as baits for assessments of protein interactions. The $\mathrm{Y} 2 \mathrm{H}$ results revealed that OsCSL1 interactions with OsMKK4 (Fig. 6A), and that this interaction was dependent on the presence of a full-length OsCSL1 (Fig. 6A). Further analysis of the expression of OsMKK genes in csl 1 mutant and WT plants revealed no significant differences in the expression of OsMKK3, OsMKK5, OsMKK6, or OsMKK10-2 between WT and the csl1 mutant (Fig. 6B); in contrast, a pronounced accumulation of OsMKK1 and OsMKK4 transcripts in the csl1 mutant was detected (Fig. 6B). Considering that it has been previously reported that OsMKK1 and OsMKK4 potentially establish interactions with OsMAPK3, OsMAPK4, and OsMAPK6 $[33,38]$, the expression of OsMAPK3, $O s M A P K 4$, and OsMAPK6 were further investigated, and accordingly observed that the expression of OsMAPK3s was significantly higher in the csl1 mutant than that in the WT (Fig. 6C). Taken together, these findings indicate the potential role of the MKKK22-MKK4 pathway in rice chloroplast development.

\section{Discussion}

In plants, events occurring during the early seedling phase are essential determinants of long-term growth and development [39]. In the present study, a homozygous chlorosis seedling lethality 1 (csl1) mutant was characterization using T-DNA insertion lines with a rice cultivar Zhonghua11 (O. japonica) background. csl1 seedlings were characterized by the development of yellow leaves (Fig. 1A) and occurrence of premature death after the trefoil stage. Inverse PCR analysis, used to identify DNA sequences flanking the T-DNA insertion region 
in the csl1 mutant, indicated that Os03g0703400, encoding OsMKKK22, was a strong candidate for the $c s l 1$ gene (Fig. 2A-C).

MAP3Ks are vital components of MAPK cascades, a group of signal transduction pathways that tend to be highly conserved in eukaryotic organisms [38]. Generally, MAPK cascades consist of three types of functional protein kinases, namely MAP3Ks, MAP2Ks, and MAPKs. MAP3Ks activate downstream MAP2Ks, which subsequently activate MAPKs that in turn target different cytoplasmic and nuclear effector proteins. Numerous studies have revealed that MAP3Ks are extensively involved in the generation of responses of plants to biotic and abiotic stresses, as well as hormonal signal transduction. For example, GhMAP3K4O is reportedly associated with a reduced tolerance to biotic and abiotic stresses in Nicotiana benthamiana via negative regulation of plant development [40]. Additionally, GhRaf19, a Raf-like MAPKKK gene, has been demonstrated to negatively regulate the development of tolerance to drought and salt stress in cotton, and to positively regulate the development of resistance to cold stress [41]. Notably, mutation of $c s l 1$, encoding a MAP3K protein in rice, was associated with a lethal yellow seedling phenotype, and that chlorophyll contents in both the leaf blades and sheaths of csl1 mutant seedlings at the trefoil stage were significantly lower than those in the WT (Fig. 1). To verify whether the observed csl1 mutant phenotype was associated with $M A P 3 K$, complementation assays and knockout experiments were performed, the results of which indicated that the development of the lethal yellow seedling phenotype in rice $c s l 1$ seedlings was attributable to mutation of the Os03g0703400 gene (Figs. 2 and 3).

As mentioned previously, most studies reported in this field have highlighted the roles of MAP3Ks in plant adaptation to environmental conditions and as a regulator of signaling. For example, CONSTITUTIVE TRIPLERESPONSE 1 (CTR1) and ENHANCED DISEASE RESISTANCE 1 (EDR1), two of the best-studied MAP3Ks in plants, are known to be involved in the ethylene-mediated signaling response. Recent studies have revealed that MAP3Ks play important roles in plant growth and development. In this regard, it has been observed that compared with WT plants, transgenic Arabidopsis plants expressing MAP3K $\delta 4$ are characterized by earlier bolting and more vigorous growth [42]; furthermore, overexpression of MAP3K 18 has been found to be associated with a smaller phenotype in Arabidopsis, demonstrating the promotion of senescence of the rosette leaves in transgenic plants compared with those of WT plants [43]. Nevertheless, the molecular mechanisms underlying the MAP3K-mediated regulation of plant growth and development, particularly leaf pigmentation, remain to be determined.

MAPK pathways are triggered in response to a diverse range of external stimuli [44], with the activation of the MAPK cascade leading to the phosphorylation of specific targets, including transcription factors, cytoskeletal proteins, and phospholipases [45]. In this regard, $\mathrm{Y} 2 \mathrm{H}$ assay results indicated that OsCSL1 established interactions with OsMKK4 (Fig. 6A), and the expression of $O s M K K 1$ and OsMKK4 was significantly increased in the csl1 mutant compared with that in the WT (Fig. 6B). It has previously been established that OsMKK1 is induced by salt stress, and that a high expression of this gene and its downstream target OsMAPK4 contributes to enhanced seedling survival by increasing the transcript levels of OsDREB2B and OsMYBS3 [38]. In rice, OsMKK4/SMG1 plays multiple roles, among which the OsMKK4-OsMPK3 and OsMKK4-OsMPK6 cascades are implicated in the development of rice immunity [46]; additionally, the OsMKK4-OsMPK1/OsMPK6 cascade and downstream transcription factor OsWRKY53 are involved in the rice wound response [47]. Furthermore, the findings of a recent study have revealed that the OsMKKK10-OsMKK4-OsMPK6 cascade leads to the coordination of a trade-off between grain number per panicle and grain size, and that this coordination is controlled by the negative regulator GSN1 [33], thereby indicating that MKK4 may function as a vital regulator of plant growth and development.

Chloroplasts are semi-autonomous organelles that are recognized as sites of photosynthesis in eukaryotic cells [48]. During early seedling development, proplastids are converted to chloroplasts via thylakoid biogenesis to facilitate energy fixation and metabolite production [39]. Compared with WT plants, discrete thylakoid structures were rarely detected in the chloroplasts of csl1 mutant plants (Fig. 1D), and consistently, the abnormal leaf pigmentation of these plants indicated the presence of defective chloroplasts. Several mutants displaying variegated, pale-green, and albino leaves have been identified in different plant species [10,19]. Reductions in the chlorophyll contents of leaf blades and sheaths at the trefoil stage in homozygous csl1 mutants (Fig. 1) provided evidence to indicate that the premature death of mutant seedlings could be attributable to abnormal chloroplast development and reductions in chlorophyll biosynthesis.

In higher plants, chloroplast differentiation and development involves the following three principal steps: (i) the activation of plastid DNA synthesis, (ii) an increase in chloroplast number, and (iii) synthesis of the photosynthetic apparatus. Based on the assumption that abnormal chloroplast development during the early stages of plant growth could be attributable to the suppression 
of expression of chlorophyll biosynthetic, plastid, and nuclear genes, expression patterns of a series of chloroplast-related genes were analyzed, and accordingly found that the expression of genes involved in chlorophyll biosynthesis and chloroplast development, including those of CBGs, PEPs, NEPs and NECGs, was significantly suppressed in the csl1 mutant. Considering that the csl1 mutant exhibits a seedling lethal phenotype (Fig. 5), and that the chlorophyll content of mutant seedlings is lower than that in the WT plants (Fig. 1), suggesting that observed defects in pigmentation and chloroplast development are associated with the repression of chloroplastassociated genes [39].

\section{Conclusions}

In summary, the findings of this study provide evidence to indicate that OsCSL1 encodes a MAPK3 protein necessary for chloroplast development. Furthermore, OsCSL1 may demonstrate function by regulating the expression of multiple chloroplast synthesis-related genes, thereby affecting their functions. Thus, mutation of the OsCSL1 gene will result in wide-ranging defects, including severely disrupted chloroplasts containing accumulated starch granules and chlorotic seedlings observed in this study (Fig. 6D). Considering that most previous studies reported on MAPK cascades in plants have focused on their role in the development of immunity and elicitation of stress responses, characterization of OsCSL1 will contribute to enhancing currently limited understanding of the essential roles of MAPKs during chloroplast development.

\section{Materials and methods}

\section{Plant materials and growth conditions}

The rice (Oryza sativa L.) chlorosis seedling lethality 1 (csl1) mutant, initially identified in a T-DNA insertion population with a Zhonghua11 (japonica) background, was kindly provided by Dr. Jingliu Zhang (Shanghai Institute of Plant Physiology and Ecology, Chinese Academy of Sciences). Over 3000 rice plants harboring T-DNA possessing a Ds element were generated via Agrobacterium tumefaciens-mediated transformation by Zhang and colleagues [49]. Wild-type (Zhonghua11) and csl1 mutant plants were grown in the paddy field of South China Agricultural University, Guangzhou, China $\left(23.16^{\circ}\right.$ $\mathrm{N}, 113.23^{\circ} \mathrm{E}$; subtropical climate) during the early (from late February to early July) and late (from middle July to late October).

\section{Insertion site analysis}

Inverse polymerase chain reaction (IPCR) was performed to isolate the sequences flanking the aforementioned T-DNA region. The nested primers used for the right and left sites of the T-DNA were $\mathrm{C} 1$ and $\mathrm{C} 2$, and $\mathrm{H} 1$ and $\mathrm{H} 2$, respectively (Fig. S2). Genomic DNA digestion was initially performed using HindIII. The primers used for screening the T-DNA insertion locus were $48800+$ and 5TF1 for the left site, and 49651 and 5TR2 for the right site, the sequences of which have been listed in Supplementary Table 1.

\section{Yeast two-hybrid (Y2H) assays}

To obtain yeast competent cells, single colonies of Y2HGold $(\phi 2-3 \mathrm{~mm})$ were dispersed in $1 \mathrm{~mL}$ of YPDA and then transferred to a $50-\mathrm{mL}$ tube containing $10 \mathrm{~mL}$ YPDA. Cell suspensions were incubated at $30^{\circ} \mathrm{C}$ for $16-18$ $\mathrm{h}$ under shaking conditions at $250 \mathrm{rpm}$, until an $\mathrm{OD}_{600}$ value $>1.5$ was obtained. Overnight cultures were subsequently centrifuged at 3,000 rpm for $5 \mathrm{~min}$ at room temperature, and the resulting supernatant was discarded. Following the addition of $10 \mathrm{~mL}$ of fresh YPDA, the tube containing the cell pellet was vortexed vigorously for 5 min to disperse cell clumps; subsequently, incubation was performed at $30^{\circ} \mathrm{C}$ for 3 to $4 \mathrm{~h}$ under shaking conditions at $250 \mathrm{rpm}$ until an $\mathrm{OD}_{600}$ value ranging from 0.4 0.6 was achieved. After further centrifugation at 3,000 $\mathrm{rpm}$ for $5 \mathrm{~min}$ at room temperature, the supernatant was discarded and the cell pellet was resuspended with 1.5 $\mathrm{mL}$ of $1 \times \mathrm{TE}$. To this cell suspension, $0.1 \mu \mathrm{g}$ of each plasmid DNA and $100 \mu \mathrm{g}$ carrier DNA were in a fresh $1.5-\mathrm{mL}$ tube, followed by addition of $0.1 \mathrm{~mL}$ of yeast competent cells and $0.6 \mathrm{~mL}$ of PEG/LiAc solution (PEG3350 40\%, $0.01 \mathrm{M}$ Tris-HCL, $1 \mathrm{mM}$ EDTA, and $0.1 \mathrm{M} \mathrm{LiAc}, \mathrm{pH}$ 7.5). This mixture was then incubated at $30^{\circ} \mathrm{C}$ for $30 \mathrm{~min}$ under shaking conditions at $200 \mathrm{rpm}$, followed by the addition of $70 \mu \mathrm{L}$ of DMSO and thorough mixing. The cells were then subjected to heat shock by incubating in a $42^{\circ} \mathrm{C}$ water bath for $15 \mathrm{~min}$, followed by incubation on ice for $2 \mathrm{~min}$. Following centrifugation for $30 \mathrm{~s}$ at 13000 $\mathrm{rpm}$ and room temperature, the supernatant was discarded and the pelleted cells were resuspended in $200 \mu \mathrm{L}$ of $1 \times \mathrm{TE}$. Aliquots $(100 \mu \mathrm{L})$ of this cell suspension were spread on SD/-Leu/-Trp and SD/-Leu/-Trp/-His/-Ade agar plates, respectively, followed by incubation at $30^{\circ} \mathrm{C}$ until the development of colonies was achieved.

\section{Determination of chlorophyll contents}

Chlorophyll content determination experiments were conducted in accordance with the methods described by Zhang et al. (2016) [10], with slight modifications. Fresh leaf samples were chopped using scissors, and approximately $0.1 \mathrm{~g}$ of the cut fresh leaf material was incubated with $2 \mathrm{~mL}$ of extraction buffer (ethanol: propanol: $\mathrm{H}_{2} \mathrm{O}$, 4.5:4.5:1 $\mathrm{v} / \mathrm{v} / \mathrm{v}$ ) in a 10-mL Eppendorf tube for $12 \mathrm{~h}$ in the dark at $4^{\circ} \mathrm{C}$, until all leaf samples presented with white 
coloration. Using the extraction buffer as a blank control, the maximum absorption of extracts was determined spectrophotometrically at $645 \mathrm{~nm}$ and $663 \mathrm{~nm}$. Chlorophyll contents were calculated using the following equations: Chlorophyll $a=\left(12.72 \mathrm{~A}_{663}-2.59 \mathrm{~A}_{645}\right) \times \mathrm{V} / \mathrm{W} \times$ 1000

Chlorophyll $b=\left(22.88 \mathrm{~A}_{645}-4.67 \mathrm{~A}_{663}\right) \times \mathrm{V} / \mathrm{W} \times 1000$

Total chlorophyll $=\left(20.29 \mathrm{~A}_{645}+8.05 \mathrm{~A}_{663}\right) \times \mathrm{V} / \mathrm{W} \times 1000$

\section{Electron microscopy}

The second leaves of the wild-type and csl1 the mutant seedlings were fixed using $2.5 \%$ glutaraldehyde for $24 \mathrm{~h}$ at $4^{\circ} \mathrm{C}$. Thereafter, following three washes with $0.1 \mathrm{M}$ PBS, the leaves were subjected to gradient dehydration using $30,50,70,80,90$, and $100 \%$ acetone solution at room temperature, followed by the incubation of leaves in each concentration for $15 \mathrm{~min}$. Resin penetration was then performed using an acetone: Epon812 mixture gradient $(5: 1,3: 1,1: 1,1: 3$, and 1:5 v/v), after which the leaves were incubated in pure Epon812 for $12 \mathrm{~h}$. The samples were subsequently embedded in paraffin and 60-80-nm thin sections were obtained using a microtome. The sections were subjected to staining with $2 \%$ uranyl acetate and were observed using a transmission electron microscope system (Leica EM UC6).

\section{RNA extraction and reversion-transcription quantitative PCR(RT-qPCR)}

Total RNA extraction was conducted using the TRIzol ${ }^{\circledR}$ Reagent (Invitrogen, USA) according to the manufacturer's instructions. First-strand cDNA synthesis from 2 $\mu \mathrm{g}$ of the extracted RNA (pre-treated with DNase I) was performed using ReverTra Ace ${ }^{\circledR}$ (TOYOBO, Japan) in $20-\mu \mathrm{L}$ reaction systems. The reverse transcription products thus obtained were used as templates for quantitative real-time PCR (RT-qPCR) performed using the CFX ConnectTM real-time PCR system (Bio-Rad) with TB Green $^{\text {TM }}$ Premix Ex Taq ${ }^{\text {TM }}$ II (Tli RNaseH Plus) (Takara, Japan) according to the manufacturer's instructions. Amplification conditions were $95{ }^{\circ} \mathrm{C}$ for $2 \mathrm{~min}$, followed by 40 cycles at $95{ }^{\circ} \mathrm{C}$ for $10 \mathrm{~s}$ and $60^{\circ} \mathrm{C}$ for $30 \mathrm{~s}$. Standard curves were obtained from $\mathrm{Ct}$ values and log concentration, and amplification efficiencies (E) were calculated according to the equation: $\mathrm{E}=10$ ( -1 /slope) -1 [50]. The PCR efficiency of all primers was $\geq 90 \%$ (Table S2), and PCR products were sequenced to evaluate primer specificity. The reference gene was selected from rice $A C T I N$, $\beta-T U B, G A P D H, 18 S-r R N A, U B C, U B Q 5$ and eIF- $4 \alpha$. $U B C$ gene was used as a reference after being identified using geNorm software [50]. The $2^{-\Delta \Delta C t}$ analysis method was used to measure the relative expression levels. Each target gene was calibrated according to the WT sample. The primer sequences used in RT-qPCR, in accordance with the MIQE guidelines [51], are listed in Supplemental Table 2.

\section{Plasmid construction and transformation}

To generate transgenic OsCSL1 complementation lines, a $\sim 9-\mathrm{kb}$ DNA fragment (extending from $\sim 2.5 \mathrm{~kb}$ upstream of ATG to $\sim 1 \mathrm{~kb}$ downstream of TGA) was amplified from $\mathrm{ZH} 11$ and was then cloned into a binary vector comprising a pCAMBIA2300 backbone to yield a complementary vector. To produce OsCSL1 knockout transgenic lines, CRISPR/Cas9-induced genome editing technique was utilized. sgRNA expression cassette driven by the Zea mays U6a promoter was constructed, which was then assembled into a pYLCRISPR/Cas9Pubi$\mathrm{H}$ vector as per methods previously described [52]. Upon verification via sequencing, the resultant vector was transformed into the Agrobacterium tumefaciens strain EHA105 for subsequent rice transformation. To examine the subcellular localization of the OsCSL1 protein, the pCAMBIA1300-Ubi-OsCSL1-GFP construct was generated, whereas for yeast two-hybrid $(\mathrm{Y} 2 \mathrm{H})$ analyses, different length segments of CSL1 and OsMKK4 were amplified using the corresponding primers. Segments of CSL1 were introduced into pGBKT7 at EcoRI and BamHI sites, and OsMKK4 was inserted into pGADT7 subjected to digestion with NdeI and BamHI. The corresponding primers used for amplification are listed in Supplementary Table 2.

\section{Subcellular localization}

Rice protoplasts were isolated from 1-2-week-old rice seedlings grown on $1 / 2$ MS medium, as per methods previously described by Zhang et al. [53] with minor modifications. The stems of seedlings were cut into 0.5$\mathrm{mm}$ strips using a scalpel and were submerged in $0.6 \mathrm{M}$ mannitol in the dark for $20 \mathrm{~min}$. Subsequently, mannitol used herein was replaced with an enzyme solution $(1.5 \%$ Cellulase RS, 0.75\% Macerozyme R-10, 0.6 M mannitol, $10 \mathrm{mM} \mathrm{MES}$ at $\mathrm{pH} 5.7,10 \mathrm{mM} \mathrm{CaCl}$, and $0.1 \% \mathrm{BSA}$ ), prepared immediately prior to use, and the stem trips were subject to digestion in the dark under shaking conditions $(60 \mathrm{rpm})$ for $6 \mathrm{~h}$. The protoplasts thus obtained were filtered through $40-\mu \mathrm{m}$ nylon meshes into a $50-\mathrm{mL}$ centrifuge tube and were centrifuged at $1,500 \mathrm{rpm}$ to collect the protoplast pellet. After discarding the supernatant, the pellet was resuspended in $2 \mathrm{~mL} \mathrm{W5}$ solution $\left(154 \mathrm{mM} \mathrm{NaCl}, 125 \mathrm{mM} \mathrm{CaCl}_{2}, 5 \mathrm{mM} \mathrm{KCl}\right.$, and $2 \mathrm{mM}$ MES, $\mathrm{pH}$ 5.7). The protoplast preparations were then transferred to several 2-mL centrifugal tubes, following which they were subjected to washing steps three times 
with W5 and were centrifuged at 1,500 rpm. The resultant pellet was resuspended in MMG solution (0.4 M mannitol, $15 \mathrm{mM} \mathrm{MgCl}_{2}$, and $4 \mathrm{mM} \mathrm{MES,} \mathrm{pH} \mathrm{5.7).} \mathrm{For}$ PEG-mediated transfection, $10 \mu \mathrm{g}$ of total plasmid DNA was mixed with $100 \mu \mathrm{L}$ of the protoplast preparation, and an equal volume of PEG solution [40\% w/v PEG 4000 (Fluka), $0.2 \mathrm{M}$ mannitol and $0.1 \mathrm{M} \mathrm{CaCl}_{2}$ ]. The mixture was then incubated in the dark for $15 \mathrm{~min}$ at $25^{\circ} \mathrm{C}$, after which, the protoplasts were collected via centrifugation at 1,500 rpm for $3 \mathrm{~min}$ and were then resuspended in $1 \mathrm{~mL}$ of the WI solution $(0.5 \mathrm{M}$ mannitol, $20 \mathrm{mM} \mathrm{KCl}$, and $4 \mathrm{mM}$ MES, pH 5.7). After incubation at $25^{\circ} \mathrm{C}$ for 16 $\mathrm{h}$, the protoplasts were examined using a confocal laser scanning microscope (Leica TCS SP8).

\section{Abbreviations}

MAPK: Mitogen-activated protein kinase; cs/1: Chlorosis seedling lethality 1; ZH11: Zhonghua11; MAPK: Mitogen-activated protein kinase; MKKK22: MAPK kinase kinase22; ER: Endoplasmic reticulum; CRISPR: Clustered regularly interspaced short palindromic repeats; CBGs: Chlorophyll biosynthetic genes; PEPs: Plastid-encoded RNA polymerases; NEPs: Nuclear-encoded RNA polymerase; NECGs: Nuclear-encoded chloroplast genes; PPR: Pentatricopeptide repeat protein; TEM: Transmission electron microscopy; IPCR: Inverse polymerase chain reaction; GFP: Green fluorescent protein; $Y 2 \mathrm{H}$ : Yeast two-hybrid.

\section{Supplementary Information}

The online version contains supplementary material available at https://doi. org/10.1186/s12870-021-03404-9.

Additional file 1: Figure S1. Chlorophyll contents and phenotypes of the wild-type and heterozygous cs/1 lines. Figure S2. Inverse polymerase chain reaction (IPCR) was performed to isolate sequences flanking the OsCSL1 T-DNA. Figure S3. Comparison of the predicted (upper) and amplified (lower) OsCSL1 protein products.

Additional file 2: Table S1. Segregation ratio of cs/1 heterozygotes. Table S2. The primers used in this study

\section{Acknowledgements}

We thank Dr. Jingliu Zhang (Shanghai Institute of Plant Physiology and Ecology, Chinese Academy of Sciences) for providing the rice cs/1 mutant.

\section{Authors' contributions}

$J \mathrm{~L}, \mathrm{QZ}$, and ZZ designed the project; $J \mathrm{~L}, \mathrm{QZ}$, and $Y L$ performed the experiments; JL, QZ, JZ, WW, and ZZ analyzed and interpreted the data; JL and QZ prepared Figures 1, 2, and 3; JZ,WW, and ZZ prepared Figures 4 and 5; and $J L$, WW, and ZZ wrote the manuscript. All authors have read and approved the final version of the manuscript.

\section{Funding}

This study was supported by the Key-Area Research and Development Program of Guangdong Province (2018B020202012 and 2020B020219004) and the Natural Science Foundation of China (31671645). The funding agencies providing financial support for this research project played no role in the design of the study, collection and analysis of data, or writing of the manuscript.

\section{Availability of data and materials}

The datasets used and/or analyzed during the current study will be available from the corresponding authors upon reasonable request.

\section{Declarations}

Ethics approval and consent to participate

Not applicable

\section{Consent for publication}

Not applicable

\section{Competing interests}

The authors declare that they have no competing interests.

Received: 11 June 2021 Accepted: 17 December 2021

Published online: 06 January 2022

\section{References}

1. Waters MT, Langdale JA. The making of a chloroplast. EMBO J. 2009:28(19):2861-73.

2. Yang $Y, X u$ J, Huang L, Leng $Y$, Dai L, Rao Y, et al. PGL, encoding chlorophyllide a oxygenase 1, impacts leaf senescence and indirectly affects grain yield and quality in rice. J Exp Bot. 2016;67(5):1297-310.

3. Mei J, Li F, Liu X, Hu G, Fu Y, Liu W. Newly identified CSP41b gene localized in chloroplasts affects leaf color in rice. Plant Sci. 2017:256:39-45.

4. Tominaga J, Mizutani H, Horikawa D, Nakahara Y, Takami T, Sakamoto W, et al. Rice CYO1, an ortholog of Arabidopsis thaliana cotyledon chloroplast biogenesis factor AtCYO1, is expressed in leaves and involved in photosynthetic performance. J Plant Physiol. 2016:207:78-83.

5. Kamau PK, Sano S, Takami T, Matsushima R, Maekawa M, Sakamoto W. A Mutation in GIANT CHLOROPLAST encoding a PARC6 homolog affects spikelet fertility in rice. Plant Cell Physiol. 2015;56(5):977-91.

6. Su N, Hu ML, Wu DX, Wu FQ, Fei GL, Lan Y, et al. Disruption of a rice pentatricopeptide repeat protein causes a seedling-specific albino phenotype and its utilization to enhance seed purity in hybrid rice production. Plant Physiol. 2012;159(1):227-38.

7. Lin D, Gong X, Jiang Q, Zheng K, Zhou H, Xu J, et al. The rice ALS3 encoding a novel pentatricopeptide repeat protein is required for chloroplast development and seedling growth. Rice (NY). 2015;8:17.

8. Wu L, Wu J, Liu Y, Gong X, Xu J, Lin D, et al. The rice pentatricopeptide repeat gene TCD10 is needed for chloroplast development under cold stress. Rice (NY). 2016;9(1):67.

9. Tang J, Zhang W, Wen K, Chen G, Sun J, Tian Y, et al. OsPPR6, a pentatricopeptide repeat protein involved in editing and splicing chloroplast RNA, is required for chloroplast biogenesis in rice. Plant Mol Biol. 2017;95(4-5):345-57.

10. Zhang Z, Tan J, Shi Z, Xie Q, Xing Y, Liu C, et al. Albino Leaf 1 that encodes the sole octotricopeptide repeat protein is responsible for chloroplast development in rice. Plant Physiol. 2016:325-2016.

11. Liu C, Zhu H, Xing Y, Tan J, Chen X, Zhang J, et al. Albino Leaf 2 is involved in the splicing of chloroplast group I and II introns in rice. J Exp Bot. 2016;67(18):5339-47.

12. Li Q, Zhu FY, Gao X, Sun Y, Li S, Tao Y, et al. Young Leaf Chlorosis 2 encodes the stroma-localized heme oxygenase 2 which is required for normal tetrapyrrole biosynthesis in rice. PLANTA. 2014;240(4):701-12.

13. Cao P, Ren Y, Liu X, Zhang T, Zhang P, Xiao L, et al. Purine nucleotide biosynthetic gene GARS controls early chloroplast development in rice (Oryza sativa L.). Plant Cell Rep. 2018.

14. Yagi Y, Shiina T. Recent advances in the study of chloroplast gene expression and its evolution. Front Plant Sci. 2014;5:61.

15. Hedtke B, Borner T, Weihe A. Mitochondrial and chloroplast phage-type RNA polymerases in Arabidopsis. Science. 1997;277(5327):809-11.

16. Yu QB, Huang C, Yang ZN. Nuclear-encoded factors associated with the chloroplast transcription machinery of higher plants. Front Plant Sci. 2014;5:316

17. Lerbs-Mache S. Function of plastid sigma factors in higher plants: regulation of gene expression or just preservation of constitutive transcription? Plant Mol Biol. 2011;76(3-5):235-49.

18. Hricova A, Quesada V, Micol JL. The SCABRA3 nuclear gene encodes the plastid RpoTp RNA polymerase, which is required for chloroplast 
biogenesis and mesophyll cell proliferation in Arabidopsis. Plant Physiol. 2006;141(3):942-56.

19. Zhou K, Ren Y, Zhou F, Wang Y, Zhang L, Lyu J, et al. Young Seedling Stripe1 encodes a chloroplast nucleoid-associated protein required for chloroplast development in rice seedlings. Planta. 2017;245(1):45-60.

20. Pitzschke A. Modes of MAPK substrate recognition and control. Trends Plant Sci. 2015;20(1):49-55.

21. Xu J, Zhang S. Mitogen-activated protein kinase cascades in signaling plant growth and development. Trends Plant Sci. 2015;20(1):56-64.

22. Shen X, Liu H, Yuan B, Li X, Xu C, Wang S. OsEDR1 negatively regulates rice bacterial resistance via activation of ethylene biosynthesis. Plant Cell Environ. 2011;34(2):179-91.

23. Kim JA, Cho K, Singh R, Jung $Y H$, Jeong $S H$, Kim SH, et al. Rice OsACDR1 (Oryza sativa accelerated cell death and resistance 1 ) is a potential positive regulator of fungal disease resistance. Mol Cells. 2009;28(5):431-9.

24. Shin HY, You MK, Jeung JU, Shin JS. OsMPK3 is a TEY-type rice MAPK in Group C and phosphorylates OsbHLH65, a transcription factor binding to the E-box element. Plant Cell Rep. 2014;33(8):1343-53.

25. Hu J, Zhou J, Peng X, Xu H, Liu C, Du B, et al. The Bphi008a gene interacts with the ethylene pathway and transcriptionally regulates MAPK genes in the response of rice to brown planthopper feeding. Plant Physiol. 2011;156(2):856-72.

26. Lee SK, Kim BG, Kwon TR, Jeong MJ, Park SR, Lee JW, et al. Overexpression of the mitogen-activated protein kinase gene OsMAPK33 enhances sensitivity to salt stress in rice (Oryza sativa L.). J Biosci. 2011;36(1):139-51.

27. Rao KP, Vani G, Kumar K, Wankhede DP, Misra M, Gupta M, et al. Arsenic stress activates MAP kinase in rice roots and leaves. Arch Biochem Biophys. 2011;506(1):73-82.

28. Singh P, Sinha AK. A positive feedback loop governed by SUB1A1 interaction with MITOGEN ACTIVATED PROTEIN KINASE 3 imparts submergence tolerance in rice. Plant Cell. 2016;28(5):1127.

29. Tian X, Li X, Zhou W, Ren Y, Wang Z, Liu Z, et al. Transcription factor OsWRKY53 positively regulates brassinosteroid signaling and plant architecture. Plant Physiol. 2017;175(3):1337-49.

30. Wang SH, Lim JH, Kim SS, Cho SH, Yoo SC, Koh HJ, et al. Mutation of SPOTTED LEAF3 (SPL3) impairs abscisic acid-responsive signalling and delays leaf senescence in rice. J Exp Bot. 2015;66(22):7045-59.

31. Yuan B, Shen X, Li X, Xu C, Wang S. Mitogen-activated protein kinase OsMPK6 negatively regulates rice disease resistance to bacterial pathogens. Planta. 2007;226(4):953-60.

32. Shen $X$, Yuan B, Liu H, Li X, Xu C, Wang S. Opposite functions of a rice mitogen-activated protein kinase during the process of resistance against Xanthomonas oryzae. Plant J. 2010;64(1):86-99.

33. Guo T, Chen K, Dong NQ, Shi CL, Ye WW, Gao JP, et al. GRAIN SIZE AND NUMBER1 negatively regulates the OsMKKK10-OsMKK4-OsMPK6 cascade to coordinate the trade-off between grain number per panicle and grain size in rice. Plant Cell. 2018;30(4):871-88.

34. Xu R, Duan P, Yu H, Zhou Z, Zhang B, Wang R, et al. Control of grain size and weight by the OsMKKK10-OsMKK4-OsMAPK6 signaling pathway in rice. Mol Plant. 2018;11(6):860-73.

35. Nelson BK, Cai X, Nebenfuhr A. A multicolored set of in vivo organelle markers for co-localization studies in Arabidopsis and other plants. Plant J. 2007:51(6):1126-36.

36. Lee JS, Huh KW, Bhargava A, Ellis BE. Comprehensive analysis of proteinprotein interactions between Arabidopsis MAPKs and MAPK kinases helps define potential MAPK signalling modules. Plant Signal Behav. 2008;3(12):1037-41.

37. Popescu SC, Popescu GV, Bachan S, Zhang Z, Gerstein M, Snyder M, et al. MAPK target networks in Arabidopsis thaliana revealed using functional protein microarrays. Genes Dev. 2009;23(1):80-92.

38. Wang $F$, Jing W, Zhang W. The mitogen-activated protein kinase cascade MKK1-MPK4 mediates salt signaling in rice. Plant Sci. 2014;227:181-9.

39. Kwon KC, Cho MH. Deletion of the chloroplast-localized AtTerC gene product in Arabidopsis thaliana leads to loss of the thylakoid membrane and to seedling lethality. Plant J. 2008:55(3):428-42.

40. Chen X, Wang J, Zhu M, Jia H, Liu D, Hao L, et al. A cotton Raf-like MAP3K gene, GhMAP3K40, mediates reduced tolerance to biotic and abiotic stress in Nicotiana benthamiana by negatively regulating growth and development. Plant Sci. 2015;240:10-24.

41. Jia H, Hao L, Guo X, Liu S, Yan Y, Guo X. A Raf-like MAPKKK gene, GhRaf19, negatively regulates tolerance to drought and salt and positively regulates resistance to cold stress by modulating reactive oxygen species in cotton. Plant Sci. 2016;252:267-81.

42. Sasayama D, Matsuoka D, Oka M, Shitamichi N, Furuya T, Azuma T, et al. MAP3KS4, an Arabidopsis Raf-like MAP3K, regulates plant growth and shoot branching. Plant Biotechnol-Nar. 2011;28(5):463-70.

43. Matsuoka D, Yasufuku T, Furuya T, Nanmori T. An abscisic acid inducible Arabidopsis MAPKKK, MAPKKK18 regulates leaf senescence via its kinase activity. Plant Mol Biol. 2015;87(6):565-75.

44. Teige M, Scheikl E, Eulgem T, Doczi R, Ichimura K, Shinozaki K, et al. The MKK2 pathway mediates cold and salt stress signaling in Arabidopsis. Mol Cell. 2004;15(1):141-52.

45. Danquah A, de Zelicourt A, Colcombet J, Hirt H. The role of ABA and MAPK signaling pathways in plant abiotic stress responses. Biotechnol Adv. 2014;32(1):40-52.

46. Kishi-Kaboshi M, Okada K, Kurimoto L, Murakami S, Umezawa T, Shibuya N, et al. A rice fungal MAMP-responsive MAPK cascade regulates metabolic flow to antimicrobial metabolite synthesis. Plant $\mathrm{J}$. 2010;63(4):599-612

47. Yoo SJ, Kim SH, Kim MJ, Ryu CM, Kim YC, Cho BH, et al. Involvement of the OsMKK4-OsMPK1 cascade and its downstream transcription factor OsWRKY53 in the wounding response in rice. Plant Pathol J. 2014;30(2):168-77.

48. Allen JF, de Paula WB, Puthiyaveetil S, Nield J. A structural phylogenetic map for chloroplast photosynthesis. Trends Plant Sci. 2011;16(12):645-55.

49. Wang J, Li L, Wan X, An L, Zhang J. Distribution of T-DNA carrying a Ds element on rice chromosomes. Sci China C Life Sci. 2004;47(4):322-31.

50. Kidd M, Nadler B, Mane S, Eick G, Malfertheiner M, Champaneria M, et al. GeneChip, geNorm, and gastrointestinal tumors: novel reference genes for real-time PCR. Physiol Genomics. 2007;30(3):363-70.

51. Bustin SA, Benes V, Garson JA, Hellemans J, Huggett J, Kubista M, et al. The MIQE guidelines: minimum information for publication of quantitative real-time PCR experiments. Clin Chem. 2009;55(4):611-22.

52. MaX, Liu YG. CRISPR/Cas9-based multiplex genome editing in monocot and dicot plants. Curr Protoc Mol Biol. 2016;115:31-6.

53. Zhang Y, Su J, Duan S, Ao Y, Dai J, Liu J, et al. A highly efficient rice green tissue protoplast system for transient gene expression and studying light/chloroplast-related processes. Plant Methods. 2011;7(1):30.

\section{Publisher's Note}

Springer Nature remains neutral with regard to jurisdictional claims in published maps and institutional affiliations.

Ready to submit your research? Choose BMC and benefit from:

- fast, convenient online submission

- thorough peer review by experienced researchers in your field

- rapid publication on acceptance

- support for research data, including large and complex data types

- gold Open Access which fosters wider collaboration and increased citations

- maximum visibility for your research: over $100 \mathrm{M}$ website views per year

At BMC, research is always in progress.

Learn more biomedcentral.com/submissions 